\title{
Prospective association between fasting NEFA and type 2 diabetes: impact of post-load glucose
}

\author{
D. Il'yasova • F. Wang • R. B. D'Agostino Jr • \\ A. Hanley $\cdot$ L. E. Wagenknecht
}

Received: 11 September 2009 / Accepted: 16 December 2009/Published online: 9 February 2010

(C) Springer-Verlag 2010

\begin{abstract}
Aims/hypothesis Elevated fasting NEFAs are thought to promote type 2 diabetes. Three prospective studies support this concept, showing increased diabetes risk associated with fasting NEFA. However, these prospective associations may be confounded by strong cross-sectional correlations between fasting NEFA and metabolic predictors of diabetes. To examine this assumption, we used cohort data from the Insulin Resistance Atherosclerosis Study (IRAS). Methods Within the IRAS cohort $(n=902,145$ incident cases), we examined nine metabolic variables for their confounding effect on the fasting NEFA-diabetes association: $2 \mathrm{~h}$ glucose; fasting plasma glucose; body mass index; waist circumference; waist-to-hip ratio; weight; insulin sensitivity $\left(S_{\mathrm{I}}\right)$; fasting insulin; and acute insulin response. We compared odds ratios for fasting NEFA ( $\log _{e}$ transformed and adjusted for age, sex, ethnicity and clinic) before and after inclusion of each metabolic variable into a logistic regression model.

Results Three variables ( $2 \mathrm{~h}$ glucose, BMI and $S_{\mathrm{I}}$ ) crosssectionally correlated with fasting NEFA $(r \geq 0.1, p<0.05)$. Unadjusted for metabolic predictors, fasting NEFA levels were positively associated with diabetes risk: OR 1.37
\end{abstract}

D. Il'yasova $(\bowtie) \cdot F$. Wang

Department of Community and Family Medicine,

Prevention Research Division, Duke University Medical Center,

Box 2949, Durham, NC 27710, USA

e-mail: dora.ilyasova@duke.edu

R. B. D'Agostino Jr · L. E. Wagenknecht

Public Health Sciences,

Wake Forest University School of Medicine,

Winston-Salem, NC, USA

A. Hanley

Department of Nutritional Sciences and Medicine and Dalla Lana

School of Public Health, University of Toronto,

Toronto, ON, Canada
(95\% CI 0.87-2.15) per unit on a log scale. All metabolic variables except AIR showed confounding. Inclusion of $2 \mathrm{~h}$ glucose reversed the positive association (OR 0.50 [95\% CI $0.30-0.82]$ ), whereas other predictors reduced the association to the null. The final model included the variables correlated with baseline fasting NEFA ( $2 \mathrm{~h}$ glucose, BMI and $S_{\mathrm{I}}$ ) and the demographic variables resulting in OR 0.47 (95\% CI 0.27-0.81).

Conclusions/interpretation Our results indicate that $2 \mathrm{~h}$ glucose strongly confounds the prospective association between fasting NEFA and diabetes; carefully adjusted fasting NEFA levels are inversely associated with diabetes risk.

Keywords Confounding · Non-esterified fatty acids . Prospective study . Type 2 diabetes

$\begin{array}{ll}\text { Abbreviations } \\ \text { AIR } & \text { Acute insulin response } \\ \text { ARIC } & \text { Atherosclerosis Risk in Communities } \\ \text { FI } & \text { Fasting insulin } \\ \text { FPG } & \text { Fasting plasma glucose } \\ \text { FSIGTT } & \begin{array}{l}\text { Frequently sampled intravenous glucose toler- } \\ \text { ance test }\end{array} \\ \text { IGT } & \text { Impaired glucose tolerance } \\ \text { IRAS } & \text { Insulin Resistance Atherosclerosis Study } \\ \text { NGT } & \text { Normal glucose tolerance } \\ S_{\text {I }} & \text { Insulin sensitivity } \\ \text { WC } & \text { Waist circumference }\end{array}$

\section{Introduction}

NEFA present the major fuel substrate for skeletal muscles during long periods between meals $[1,2]$. Because the 
fasting circulating levels of NEFA are proportional to bodyfat storage $[1,2]$, cross-sectional studies have consistently demonstrated positive correlations between fasting NEFA levels and obesity, as well as two of the sequelae of obesity, insulin resistance and glucose intolerance [1, 2]. It is unclear whether elevated NEFA levels during fasting are relevant to further deterioration of glucose homeostasis or are simply associated with metabolic type 2 diabetes predictors. Answering the question of how fasting NEFA levels relate to the development of type 2 diabetes is important because it could clarify whether fasting NEFA levels are on the causal pathway of lipotoxicity - one of the important aetiological bases for the development of type 2 diabetes.

To determine how fasting NEFA level relates to the development of type 2 diabetes requires examination of their prospective association. However, baseline crosssectional correlations between fasting NEFA and type 2 diabetes metabolic predictors can have a profound distorting effect on the prospective fasting NEFA-diabetes association. Extreme cases of distorted associations have been previously documented and are known as Simpson's paradox [3] or qualitative confounding [4]. The most common textbook example of this phenomenon is the effect of age adjustment on mortality statistics. Age is a major correlate of mortality; therefore, direct comparison of mortality rates between populations with two different age structures is deceiving. For example, comparison of mortality rates in the USA vs Venezuela shows increased mortality risk in the USA (mortality ratio 1.98 ), while age adjustment reverses the association (mortality ratio 0.78 ) [4]. Similarly, strong correlates of fasting NEFA, such as glucose intolerance, insulin sensitivity and obesity, may drive the unadjusted estimate for the prospective fasting NEFA-diabetes association.

Therefore, we hypothesised that the choice of adjustment variable may strongly influence the results of a prospective analysis. Among the previously published prospective studies on this topic, three of the four found an increased risk of glucose intolerance and/or diabetes in individuals with elevated fasting NEFA [5-7]. The fourth study, in contrast, showed the expected cross-sectional correlation between fasting NEFA level and glucose intolerance but did not confirm the positive prospective association [8]. One study compared the means for NEFA in different subgroups stratified by metabolic variables, but did not present the estimates of relative risk [8]. Three other studies presented the estimates of the relative risk from multiple models adjusting for different sets of metabolic variables, but the confounder-adjustment approaches varied and may not have optimally controlled for the important associations between fasting NEFA and other baseline type 2 diabetes predictors; also, these three studies did not present a subgroup analysis [5-7]. To examine carefully the influence of metabolic variables on the prospective association between fasting NEFA level and type 2 diabetes, we conducted a detailed analysis of the prospective fasting NEFA-diabetes association using cohort data from the well-documented Insulin Resistance Atherosclerosis Study (IRAS).

\section{Methods}

IRAS study population

The IRAS is a multicentre population-based cohort study [9] that recruited a total of 1625 men and women, 40 to 69 years of age, from four US communities from 1992 to 1993. The study recruited approximately equal numbers of persons with normal glucose tolerance, impaired glucose tolerance and type 2 diabetes, as well as equal numbers of non-Hispanic whites, Hispanics and African-Americans. In the follow-up examination conducted 5 years after recruitment, $80 \%$ of the cohort participated. The IRAS protocol was approved by local institutional review committees, and all participants gave informed consent.

\section{Definition of glucose tolerance}

Glucose tolerance was measured precisely at each examination using an oral glucose tolerance test and the World Health Organization criteria. A 75 g glucose load (Orangedex; Custom Laboratories, Baltimore, MD, USA) was administered over a period of $<10 \mathrm{~min}$. Blood was collected at 0 and $2 \mathrm{~h}$. Normal glucose tolerance was defined as fasting glucose and $2 \mathrm{~h}$ glucose $<7.77 \mathrm{mmol} / 1$ (140 mg/dl). Impaired glucose tolerance was defined as fasting glucose $<7.77 \mathrm{mmol} / \mathrm{l}(140 \mathrm{mg} / \mathrm{dl})$ and $2 \mathrm{~h}$ glucose $\geq 7.77 \mathrm{mmol} / \mathrm{l}$ $(140 \mathrm{mg} / \mathrm{dl})$ and $<11.1 \mathrm{mmol} / 1(200 \mathrm{mg} / \mathrm{dl})$. Diabetes was defined as fasting glucose $\geq 7.77 \mathrm{mmol} / 1$ (140 mg/dl) or $2 \mathrm{~h}$ glucose $\geq 11.1 \mathrm{mmol} / 1$ (200 mg/dl) or use of hypoglycaemic medications.

Measurements of metabolic variables

Clinical measures and procedures All participants fasted for $12 \mathrm{~h}$ and refrained from heavy exercise, smoking and alcohol consumption for $24 \mathrm{~h}$ before the visit. Insulin sensitivity and insulin secretion were determined using the frequently sampled intravenous glucose tolerance test (FSIGTT), with two modifications to the original protocol [10]. First, an injection of regular insulin, rather than tolbutamide, was used to ensure adequate plasma insulin levels for the accurate computation of insulin sensitivity across a broad range of glucose tolerances [11]. Second, a 
reduced sampling protocol (with 12 rather than 30 samples) was employed for efficiency, given the large number of participants [12]. Both insulin sensitivity $\left(S_{\mathrm{I}}\right)$ and acute insulin response (AIR) were calculated using mathematical modelling methods (MINMOD version 3.0 1994) [13]. The reliability of $S_{\mathrm{I}}$ and AIR calculations was demonstrated in a subsample of the IRAS cohort. The estimate of $S_{\text {I }}$ produced by the modified protocol has been validated against the gold-standard measures of insulin resistance from the hyperinsulinaemic-euglycaemic clamp technique $(r=0.55)$ [14], and AIR has been validated by others using goldstandard measures of insulin secretion from the hyperglycaemic clamp technique [15].

Anthropometric measurements included height, waist and hip circumferences (all three measured to the nearest $0.5 \mathrm{~cm}$ ) and weight (measured to the nearest $0.1 \mathrm{~kg}$ ). All measures were obtained in duplicate following a standardised protocol, and averages were used in the analysis. Body mass index, calculated as weight/height ${ }^{2}\left(\mathrm{~kg} / \mathrm{m}^{2}\right)$, was used as an estimate of overall adiposity. The average of duplicate measurements was used to calculate the waist-tohip ratio.

Laboratory measurements Glucose concentration was determined using standard methods [9]. Insulin levels were measured using the dextran-charcoal radioimmunoassay [16]. Plasma NEFA concentrations were measured colorimetrically as previously described [17].

\section{Statistical analysis}

The analytical cohort included participants $(n=902)$ who had normal or impaired glucose tolerance at baseline, had baseline measurements of fasting NEFA and participated in the follow-up examination. During the 5 year study period, 145 IRAS participants in the analytical cohort developed type 2 diabetes, while the remaining 757 participants showed normal or impaired glucose tolerance at the follow-up examination. Participant characteristics (mean [SD] for continuous variables and per cent observed for categorical characteristics) were estimated overall, by glucose tolerance status at baseline (NGT/IGT) and by type 2 diabetes converter status (yes/no). Further analyses were performed using the following steps.

Step 1 Using Spearman's rank correlation coefficients $(r)$ and the corresponding $p$ values, we examined simple bivariate correlations between baseline fasting NEFA and eight metabolic variables: $2 \mathrm{~h}$ glucose; fasting plasma glucose; BMI; waist circumference; waist-to-hip ratio; weight; insulin sensitivity $\left(S_{\mathrm{I}}\right)$; fasting insulin (FI); and acute insulin response (AIR). These correlations were examined overall and stratified by baseline glucose tolerance status (NGT/IGT). To better visualise cross-sectional relationships between fasting NEFA and $2 \mathrm{~h}$ glucose- the variable that was most strongly correlated with baseline fasting NEFA - we graphically examined the levels of fasting NEFA ( $\log _{e}$-transformed) by the quintiles of $2 \mathrm{~h}$ glucose (Fig. 1a). Further, we asked the question whether type 2 diabetes converters had higher levels of fasting NEFA at baseline; to answer this question, we graphically examined mean values of fasting NEFA ( $\log _{e}$ transformed) by converter status within each quintile of $2 \mathrm{~h}$ glucose (Fig. 1b).

Step 2 Further, to evaluate the association between fasting NEFA level and type 2 diabetes incidence, we fitted a series of unconditional logistic regression models with the development of type 2 diabetes (yes/no) as the outcome and fasting NEFA ( $\log _{e}$-transformed) as the predictor of interest using different sets of risk factors included in each
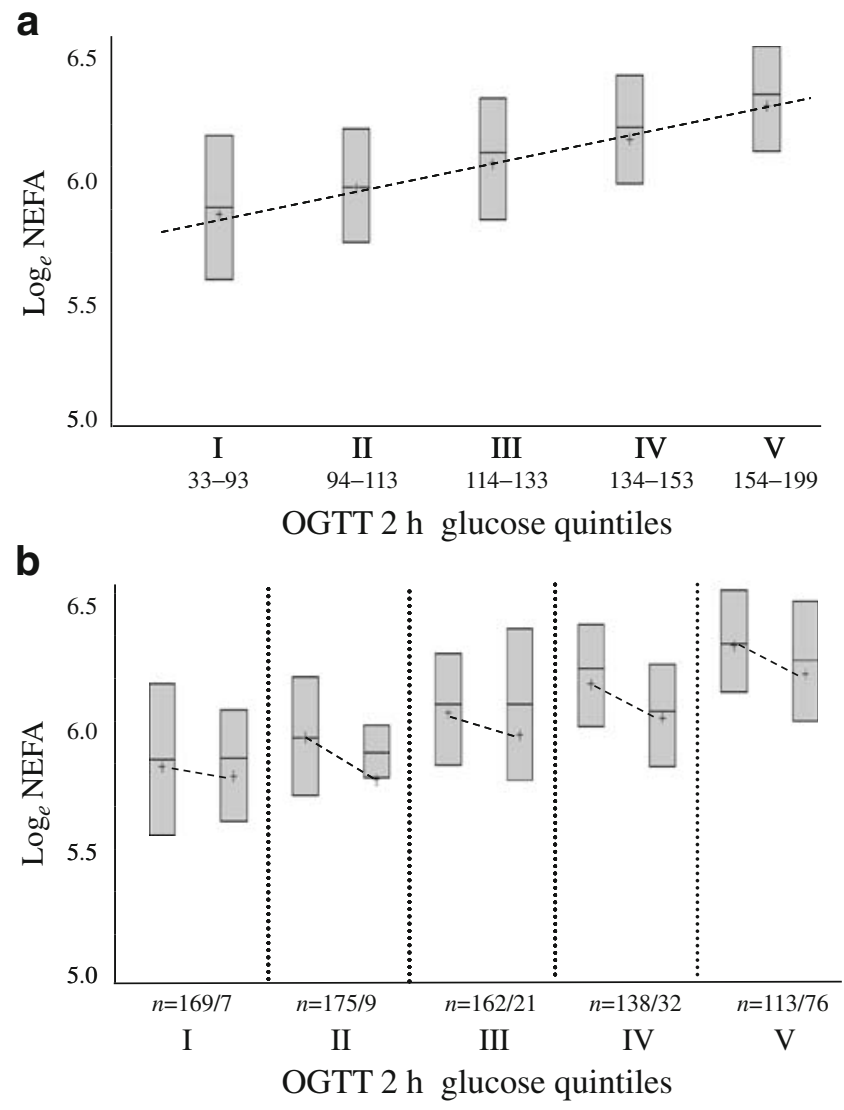

Fig. 1 Distribution of fasting NEFA levels by quintiles of OGTT $2 \mathrm{~h}$ glucose at baseline of the IRAS: the boxes indicate the range from 25th to 75th percentile; the horizontal lines within boxes depict the median values; + indicates the mean values within subgroups; the dashed lines emphasise the differences between the mean values. b For each quintile, the box on the left of each shows the values for the non-converters, while the box on the right shows the value for the converters 
model. Fasting NEFA was $\log _{e}$ transformed in these models, because its distribution was strongly right-skewed and a natural logarithmic transformation made its distribution appear more symmetric. For each model, we examined the odds ratio and the corresponding $95 \% \mathrm{CI}$ describing the relationship between fasting NEFA and the incidence of type 2 diabetes.

The first model (model 1) included fasting NEFA and demographic/study site variables: age (years), sex, ethnicity (non-Hispanic white, African-American, Hispanic) and study site. To assess whether metabolic predictors of type 2 diabetes confound the association between fasting NEFA and type 2 diabetes risk, we examined how the addition of metabolic predictors influenced the associations observed in Model 1. For each metabolic predictor, we fitted two types of model. The first included all variables in Model 1 plus the specific metabolic predictor and was fitted using all participants. The next set of models stratified the participants based on the baseline value of the metabolic predictor. For each metabolic predictor, the stratified models were based either upon using conventionally accepted categories or were based on a median split. Variables using conventionally accepted categories included $2 \mathrm{~h}$ glucose (NGT/IGT), FPG (cut-off point at $6.105 \mathrm{mmol} / \mathrm{l}[110 \mathrm{mg} / \mathrm{dl}]$ ), and BMI (normal vs [overweight + obese]). Variables using categories based on the median included WHR, weight, waist circumference, AIR, $S_{I}$ and FI.

Step 3 Based on the results from step 2, we fitted a final model using the following principles. The final model included metabolic variables that changed the strength of the association between fasting NEFA and type 2 diabetes by at least $20 \%$ : [OR (model 1)-OR (model $1+$ metabolic predictor) $] / O R$ (model 1$) \geq|0.2|]$. We based this criterion on the change-in-estimate principle suggested by Mickey and Greenland [18]. The authors suggested the criterion of $10 \%$ change in the estimate; however, they noted that this criterion was arbitrary [18]. We increased the threshold of this criterion to a $20 \%$ change to focus on more robust confounding effects. When we used this algorithm for selecting the metabolic variables to be included in the final model, we noted that some of the metabolic variables reflected similar metabolic phenomena; for example, FPG and $2 \mathrm{~h}$ glucose are used to describe individual status of glucose homeostasis. If this occurred, that is, if two (or more) metabolic variables showed important confounding $(\geq 20 \%)$ and both related to the same metabolic phenomenon, we included in the final model the one that had the stronger correlation with fasting NEFA levels at baseline. Using this approach, the final model included $\log _{e}$ (NEFA), 2 h glucose, BMI, $S_{\mathrm{I}}$, age, sex, ethnicity and study site.

Statistical analysis has been performed using SAS 9.2 (SAS Institute, Cary, NC, USA).

\section{Results}

The baseline characteristics show an ethnically diverse cohort (Table 1), with large proportions of AfricanAmericans (26.5\%) and Hispanics (33.5\%). One-third of the study population showed glucose intolerance at baseline and approximately $16 \%$ of the cohort converted to type 2 diabetes during the 5 year follow-up period.

Correlations between baseline fasting NEFA and metabolic predictors of type 2 diabetes

Fasting NEFA correlated positively with three metabolic variables $(2 \mathrm{~h}$ glucose, BMI, waist circumference and fasting insulin), and correlated negatively with insulin sensitivity $\left(S_{\mathrm{I}}\right.$; Table 2 , all participants). Correlations with other variables did not reach our statistical cut-off point $(p<0.05$; Table 2, all participants).

Distribution of fasting NEFA stratified by $2 \mathrm{~h}$ glucose levels

We selected $2 \mathrm{~h}$ glucose levels, the strongest type 2 diabetes predictor, as a stratification factor to examine the distribution of fasting NEFA levels at baseline. Fasting NEFA levels steadily but not strongly increased by quintiles of $2 \mathrm{~h}$ glucose (Fig. 1a), confirming their cross-sectional association. However, when we stratified the distributions by converter status within each quintile of $2 \mathrm{~h}$ glucose, converters did not have higher levels of fasting NEFA at baseline (Fig. 1b).

Prospective association between fasting NEFA and type 2 diabetes risk

Exploratory analysis After adjustment for demographic variables, the odds ratio of the association between NEFA and type 2 diabetes risk was 1.37 , although it was not statistically significant (Table 3 ). In fact, this adjustment did not meaningfully change the univariate association between fasting NEFA and type 2 diabetes risk: the OR for univariate association was 1.32 (95\% CI $0.86-2.02)$. However, simple stratification by glucose-tolerance status reversed the direction of the odds ratio: the overall OR was 1.37, but the subgroup-specific ORs for the NGT and IGT subgroups were 0.83 and 0.66 , respectively. This demonstrates the confounding effect of glucose-tolerance status, a major metabolic predictor of type 2 diabetes. Substitution of a continuous $2 \mathrm{~h}$ glucose variable for dichotomous glucose-tolerance status revealed an obvious inverse association between fasting NEFA and type 2 diabetes risk (OR $0.50, p<0.05)$, which persists in the NGT and IGT subgroups (OR 0.57 and 0.50 , respectively). Adjustment for other 
Table 1 Baseline characteristics of the study population

\begin{tabular}{|c|c|c|c|c|c|}
\hline Characteristic $^{\mathrm{a}}$ & All $(n=902)$ & $\begin{array}{l}\text { NGT } \\
(n=601)\end{array}$ & $\begin{array}{l}\text { IGT } \\
(n=301)\end{array}$ & $\begin{array}{l}\text { Non-converters } \\
(n=757)\end{array}$ & $\begin{array}{l}\text { Converters } \\
(n=145)\end{array}$ \\
\hline Age (years) & $54.6(8.4)$ & $53.7(8.5)$ & $56.5(8.0)$ & $54.4(8.5)$ & $55.9(7.8)$ \\
\hline Sex, female $(\%)$ & 56.5 & 53.7 & 62.1 & 56.0 & 59.3 \\
\hline \multicolumn{6}{|l|}{ Ethnicity (\%) } \\
\hline Non-Hispanic white & 40.0 & 40.8 & 38.5 & 40.6 & 37.2 \\
\hline African-American & 26.5 & 25.8 & 27.9 & 26.2 & 28.3 \\
\hline Hispanic & 33.5 & 33.4 & 33.6 & 33.3 & 34.5 \\
\hline Fasting NEFA $(\mu \mathrm{mol} / \mathrm{l})$ & $468.9(186.4)$ & $429.2(171.5)$ & $548.2(190.0)$ & $464.6(185.0)$ & $491.4(192.8)$ \\
\hline $2 \mathrm{~h}$ glucose $(\mathrm{mmol} / \mathrm{l})$ & $6.93(1.88)$ & $5.86(1.17)$ & $9.07(0.95)$ & $6.63(1.75)$ & $8.50(1.69)$ \\
\hline Fasting plasma glucose (mmol/l) & $5.47(0.62)$ & $3.63(0.549)$ & $5.81(0.61)$ & $5.38(0.57)$ & $5.93(0.67)$ \\
\hline BMI $\left(\mathrm{kg} / \mathrm{m}^{2}\right)$ & $28.4(5.6)$ & $27.4(4.8)$ & $30.5(6.5)$ & $28.0(5.3)$ & $31.2(6.4)$ \\
\hline Waist-to-hip ratio & $0.86(0.09)$ & $0.85(0.08)$ & $0.87(0.09)$ & $0.85(0.09)$ & $0.88(0.08)$ \\
\hline Waist (cm) & $90.3(12.7)$ & $88.0(11.5)$ & $94.9(13.8)$ & $89.3(12.4)$ & $95.7(13.1)$ \\
\hline Weight $(\mathrm{kg})$ & $79.8(16.9)$ & $77.6(15.5)$ & $84.2(18.7)$ & $78.6(16.3)$ & $85.8(18.9)$ \\
\hline Fasting insulin (pmol/1) & $94.1(89.1)$ & $82.7(58.1)$ & $116.7(127.7)$ & $86.2(66.9)$ & $134.9(155.5)$ \\
\hline Acute insulin response $\left(\mathrm{pmol} \mathrm{ml} \mathrm{min}^{-1} \mathrm{~min}^{-1}\right.$ ) & $489.6(489.4)$ & $555.0(524.1)$ & $359.0(380.0)$ & $529.5(506.8)$ & $277.4(308.0)$ \\
\hline Insulin sensitivity, $S_{\mathrm{I}}\left(\times 10^{-4} \mathrm{~min}^{-1} \mathrm{pmol}^{-1} \mathrm{ml}^{-1}\right)$ & $0.367(0.334)$ & $0.434(0.367)$ & $0.217(0.200)$ & $0.401(0.351)$ & $0.217(0.367)$ \\
\hline
\end{tabular}

${ }^{a}$ Mean (SD) presented for continuous variables; \% presented for categorical characteristics

metabolic predictors (except AIR) brought the OR for the association between fasting NEFA and type 2 diabetes risk from OR 1.37 closer to the null. Adjustment for AIR did not noticeably change the association from model 1 . Stratification by the baseline levels of metabolic predictors did not reveal any strong effect modification: the $p$ values for interaction between fasting NEFA and BMI, waist circumference and weight were $0.1,0.3$ and 0.3 , respectively.

Final model The results of the correlation and exploratory analyses show that $2 \mathrm{~h}$ glucose exerts the strongest confounding effect on the association between fasting
NEFA and type 2 diabetes risk. Besides $2 \mathrm{~h}$ glucose, FPG, BMI, waist circumference and $S_{\mathrm{I}}$ reduced the OR from model 1 by $30 \%, 21 \%, 21 \%$ and $26 \%$, respectively. Out of the five metabolic variables that exerted a confounding effect above the selected threshold, we retained three variables $\left(2 \mathrm{~h}\right.$ glucose, BMI, and $\left.S_{\mathrm{I}}\right)$ in the final model (Table 4). Because FPG is analogous to the $2 \mathrm{~h}$ glucose variable (reflecting glucose-tolerance status) but has a lesser confounding effect and did not correlate with fasting NEFA levels at baseline, it was not included in the model. Similarly, BMI showed a stronger crosssectional correlation with fasting NEFA at baseline than

Table 2 Baseline correlations between fasting NEFA and metabolic variables ${ }^{\mathrm{a}}$

\begin{tabular}{llll}
\hline Variable & \multicolumn{1}{l}{ Fasting NEFA } & & \\
\cline { 2 - 4 } & All participants & NGT & IGT \\
\hline 2h glucose & $0.371(<0.0001 ; 902)$ & $0.244(<0.0001 ; 601)$ & $0.170(0.003 ; 301)$ \\
FPG & $0.056(0.09 ; 902)$ & $-0.042(0.3 ; 601)$ & $-0.147(0.01 ; 301)$ \\
BMI & $0.156(<0.0001 ; 900)$ & $0.081(0.05 ; 599)$ & $0.103(0.08 ; 301)$ \\
WHR & $-0.059(0.08 ; 899)$ & $-0.080(0.05 ; 599)$ & $-0.142(0.01 ; 300)$ \\
Weight & $0.013(0.7 ; 900)$ & $-0.059(0.15 ; 599)$ & $0.013(0.8 ; 301)$ \\
Waist & $0.075(0.02 ; 899)$ & $0.001(1.0 ; 599)$ & $-0.002(1.0 ; 300)$ \\
Fasting insulin & $0.113(0.001 ; 901)$ & $0.085(0.04 ; 600)$ & $-0.013(0.8 ; 301)$ \\
AIR & $0.024(0.5 ; 878)$ & $0.067(0.1 ; 585)$ & $0.044(0.5 ; 293)$ \\
S & $-0.228(<0.0001 ; 838)$ & $-0.139(0.001 ; 560)$ & $-0.135(0.03 ; 278)$ \\
\hline
\end{tabular}

Values are Spearman correlation coefficients: $r$ ( $p$ value; number of observations)

${ }^{a}$ All the variables are in the original scale 
Table 3 Association of fasting NEFA level with the risk of diabetes - adjustment for and stratification by metabolic risk factors

Variables included in the model ${ }^{\mathrm{a}} \quad$ Stratification by baseline characteristics OR (95\% CI) (n)

\begin{tabular}{|c|c|c|c|}
\hline & & & \\
\hline & All & Subgroups & \\
\hline $\log _{e}(\mathrm{NEFA})$ (Model1) & $1.37(0.87-2.15)(902)$ & NGT $0.83(0.40-1.73)(601)$ & IGT $0.66(0.34-1.27)(301)$ \\
\hline $\log _{e}(\mathrm{NEFA}), 2 \mathrm{~h}$ glucose & $0.50(0.30-0.82)(902)$ & NGT $0.57(0.27-1.20)(601)$ & IGT $0.50(0.25-1.01)(301)$ \\
\hline $\log _{e}(\mathrm{NEFA}), \mathrm{FPG}$ & $0.97(0.60-1.55)(902)$ & $\begin{aligned} & \mathrm{FPG}<6.105 \mathrm{mmol} / \mathrm{l} \\
& 0.99(0.57-1.75)(754)\end{aligned}$ & $\begin{aligned} \mathrm{FPG} & \geq 6.105 \mathrm{mmol} / \mathrm{l} \\
0.95 & (0.37-2.45)(148)\end{aligned}$ \\
\hline $\log _{e}(\mathrm{NEFA}), \mathrm{BMI}$ & $1.08(0.69-1.71)(900)$ & $\begin{array}{l}\text { Normal }\left(\mathrm{BMI}<25.0 \mathrm{~kg} / \mathrm{m}^{2}\right) \\
0.62(0.21-1.78)(234)\end{array}$ & $\begin{array}{l}\text { Overweight/obese } \\
\left(\mathrm{BMI} \geq 25.0 \mathrm{~kg} / \mathrm{m}^{2}\right) \\
1.21(0.72-2.02)(666)\end{array}$ \\
\hline $\log _{e}(\mathrm{NEFA}), \mathrm{WHR}$ & $1.22(0.77-1.93)(899)$ & $\begin{array}{l}\text { WHR }<0.857 \text { (median) } \\
1.21(0.58-2.54)(448)\end{array}$ & $\begin{array}{l}\text { WHR } \geq 0.857 \text { (median) } \\
1.18(0.65-2.15)(451)\end{array}$ \\
\hline $\log _{e}(\mathrm{NEFA})$, waist & $1.08(0.68-1.71)(899)$ & $\begin{array}{r}\text { Waist }<89.5 \mathrm{~cm} \text { (median) } \\
0.86(0.41-1.80)(449)\end{array}$ & $\begin{array}{r}\text { Waist } \geq 89.5 \mathrm{~cm} \text { (median) } \\
1.22(0.68-2.22)(450)\end{array}$ \\
\hline $\log _{e}(\mathrm{NEFA})$, weight & $1.15(0.73-1.81)(900)$ & $\begin{array}{c}\text { Weight }<77.84 \mathrm{~kg} \text { (median) } \\
0.73(0.34-1.57)(449)\end{array}$ & $\begin{array}{c}\text { Weight } \geq 77.84 \mathrm{~kg} \text { (median) } \\
1.41(0.77-2.57)(451)\end{array}$ \\
\hline $\log _{e}(\mathrm{NEFA}), S_{\mathrm{I}}$ & $(0.60-1.70)(838)$ & $\begin{array}{l}S_{\mathrm{I}}<0.443 \times 10^{-4} \mathrm{~min}^{-1} \mathrm{pmol}^{-1} \mathrm{ml}^{-1} \\
\quad(\text { median })(0.51-1.98)(421)\end{array}$ & $\begin{array}{l}S_{\mathrm{I}} \geq 0.443 \times 10^{-4} \mathrm{~min}^{-1} \mathrm{pmol}^{-1} \mathrm{ml}^{-1} \\
(\text { median) } 0.82(0.36-1.87)(417)\end{array}$ \\
\hline $\log _{e}(\mathrm{NEFA})$, fasting insulin & $1.17(0.74-1.85)(901)$ & $\begin{array}{l}\text { Fasting insulin }<13 \mathrm{pmol} / 1 \text { (median) } \\
1.10(0.48-2.51)(433)\end{array}$ & $\begin{array}{l}\text { Fasting insulin } \geq 13 \mathrm{pmol} / 1 \\
\quad \text { (median) } 1.13(0.65-1.98)(468)\end{array}$ \\
\hline $\log _{e}(\mathrm{NEFA})$, AIR (MM) & $1.40(0.88-2.23)(878)$ & $\begin{array}{l}\mathrm{AIR}<374.42 \mathrm{pmol} \mathrm{ml}^{-1} \mathrm{~min}^{-1} \\
(\text { median) } 1.42(0.82-2.47)(438)\end{array}$ & $\begin{array}{l}\mathrm{AIR} \geq 374.42 \mathrm{pmol} \mathrm{ml}^{-1} \mathrm{~min}^{-1} \\
(\text { median) } 1.17(0.48-2.86)(440)\end{array}$ \\
\hline
\end{tabular}

${ }^{a}$ All models also included the following demographic and study variables: age, ethnicity, sex, study site

waist circumference: the correlation coefficient for NEFABMI was approximately twice that for NEFA-waist (Table 2). For this reason we chose BMI to represent adiposity in the final model.

With careful adjustment for confounders, the final model showed an inverse association between fasting NEFA and type 2 diabetes risk (OR 0.47). This association did not change meaningfully when stratified by IGT status: OR

Table 4 Final model for the association of fasting NEFA and type 2 diabetes risk

\begin{tabular}{|c|c|}
\hline Variables included in the model ${ }^{\mathrm{a}}$ & OR $(95 \% \mathrm{CI})$ \\
\hline $\log _{e}(\mathrm{NEFA})$ (1 unit on a $\log$ scale) & $0.47(0.27-0.81)$ \\
\hline $2 \mathrm{~h}$ glucose $\left(\right.$ per SD) ${ }^{\mathrm{b}}$ & $3.01(2.31-3.97)$ \\
\hline$S_{\mathrm{I}}($ per SD) & $0.70(0.47-1.00)$ \\
\hline BMI (per SD) & $1.28(1.03-1.60)$ \\
\hline Age (per SD) & $1.13(0.91-1.40)$ \\
\hline Sex (female vs male) & $1.06(0.68-1.65)$ \\
\hline \multicolumn{2}{|l|}{ Ethnicity: } \\
\hline Non-Hispanic white & Reference \\
\hline African-American & $0.81(0.43-1.51)$ \\
\hline Hispanic & $1.13(0.61-2.15)$ \\
\hline
\end{tabular}

${ }^{a}$ Study site was included in the model; there was no association with the study site

${ }^{\mathrm{b}}$ OR shows the change in the risk of type 2 diabetes associated with increase equal to 1 standard deviation
0.50 and 0.51 for participants with NGT and IGT at baseline, respectively. Because AIR is a known independent predictor of type 2 diabetes, we added this variable into the final model to examine whether the association between fasting NEFA and type 2 diabetes would be sensitive to such an addition. The OR for fasting NEFA did not change (OR $0.48,95 \%$ CI $0.27-0.85$ ). As expected, however, the AIR predicted type 2 diabetes risk independently of other risk factors (OR $0.38,95 \%$ CI $0.26-0.55$ ).

\section{Discussion}

Our analysis of the IRAS cohort data documents a classic case of confounding. To be a confounder, a suspected variable should be associated with both the main exposure of interest and the outcome. In our study, several metabolic variables were known to be independently associated with the risk of diabetes [19] and also correlated with the main effect variable-baseline fasting NEFA levels (Table 2). When adjusted for the confounders ( $2 \mathrm{~h}$ glucose, BMI, $\left.S_{\mathrm{I}}\right)$ and the demographic/study variables, fasting NEFA levels were inversely and independently associated with the risk of diabetes (final model, Table 4). The expected associations with known type 2 diabetes risk factors-positive associations with $2 \mathrm{~h}$ glucose and BMI and inverse associations with $S_{\mathrm{I}}$ and AIR-demonstrated that the analytical cohort is comparable with other study popula- 
tions and that our results are generalisable. Further, data presented in Fig. 1 suggest that fasting NEFA is not a cause but a consequence of deterioration of glucose tolerance.

In our analysis, $2 \mathrm{~h}$ glucose operated as the key confounder. This variable showed the strongest correlation with baseline fasting NEFA levels and most strongly predicted type 2 diabetes incidence. Adjustment for baseline $2 \mathrm{~h}$ glucose represents the crucial distinction between our study and two earlier studies that reported a positive fasting NEFA-diabetes association. The first, a prospective study of Pima Indians conducted by Paolisso and colleagues [7], measured glucose tolerance by the OGTT but did not adjust for baseline $2 \mathrm{~h}$ glucose; the relative risk associated with the comparison of the 90th vs 10th percentiles of fasting NEFA was 2.3 (95\% CI 1.1-4.7).

In the second study, a prospective case-control study nested in the Atherosclerosis Risk in Communities (ARIC) cohort [5], Pankow and co-investigators used FPG (instead of $2 \mathrm{~h}$ glucose) for the diagnosis of type 2 diabetes and for adjustment of the fasting NEFA-diabetes association. The study found a positive association between fasting NEFA and type 2 diabetes risk after adjustment for baseline FPG; the hazard ratio for comparing the fourth NEFA quartile with the first fasting NEFA quartile was $1.68(95 \%$ CI 1.20-2.34). Based on our findings, adjustment for FPG is not equivalent to the adjustment for $2 \mathrm{~h}$ glucose (Table 3 ), which can explain the discrepancy in the results. Besides, there was no baseline correlation between FPG and fasting NEFA in the ARIC study, indicating that in the ARIC population FPG did not confound the fasting NEFAdiabetes association. Although substitution of $2 \mathrm{~h}$ glucose by FPG is a conventional choice in many epidemiological studies and is probably well justified, some associations can be highly sensitive to such substitution.

In a 2 year study among male police employees in Paris [6], Charles and colleagues included $2 \mathrm{~h}$ glucose in their analysis. Even after adjustment for this key confounder (among several other variables), ORs associated with the increase in fasting NEFA by one standard deviation (0.12 mmol/l) were $1.3(95 \%$ CI 1.1-1.4) for converting from NGT to either IGT or type 2 diabetes [6]. However, the Paris study population seems to differ from the general population in at least two important ways: by a high rate of reversal from IGT to NGT (65\%) and no independent association between BMI and diabetes risk. Such deviations from other study populations preclude generalisation of these results to other populations.

The discrepancy between the inverse association found in our study and the positive associations reported by the Pima Indian [7] and ARIC [5] cohort studies can be explained by incomplete adjustment for confounding, while the discrepancy with the Paris study [6] is probably due to the specifics of the study population. In contrast, a fourth population-based cohort Ely study [8] stratified the analysis by baseline-IGT status as measured by the OGTT. The analysis compared means of fasting NEFAs in different subgroups without modelling relative risk with sophisticated adjustment for confounders. In the absence of multivariable modelling but stratifying by IGT status, this analysis clearly demonstrated trends similar to our findings. The study found a cross-sectional correlation between fasting NEFA and glucose intolerance with higher fasting NEFA levels in IGT participants. At the same time, those who developed diabetes or IGT did not have higher fasting NEFA levels at baseline. Taken together, our analysis and the Ely study confirm the importance of adjusting for $2 \mathrm{~h}$ glucose. Even crude adjustment by baseline IGT status disproves the hypothesis that increased fasting NEFA levels predict diabetes risk.

The striking finding that the cross-sectional correlation between fasting NEFA and type 2 diabetes risk factors is positive while the prospective association is negative can be explained by two factors: the paradoxical relationship between fat oxidation and diabetes on the one hand and on the other hand, the relationship between circulating NEFA levels and intensity of fat oxidation. In frank diabetes and obesity, fat oxidation on average is increased [20]. However, prospective metabolic studies demonstrate that high respiratory quotient, an indicator of low fat oxidation, predicts weight gain [21-26], implying that slow fat oxidation predisposes to obesity. Moreover, a number of crosssectional studies support the concept that low ability to oxidise fat is involved in metabolic deterioration. Lower levels of fat utilisation have been demonstrated in formerly obese individuals as compared with their never-obese counterparts [27-30], and reduced NEFA oxidation in skeletal muscles has been demonstrated in obese patients with type 2 diabetes compared with obese controls without diabetes [31-33]. Insulin resistance also has been shown to be inversely associated with whole-body fat oxidation in the non-diabetic offspring of type 2 diabetes patients, suggesting that genetic predisposition to type 2 diabetes involves low individual ability to oxidise fat [34]. Finally, the opposite effect of exercise training on fat oxidation (increase) [35-37] and type 2 diabetes risk (decrease) suggest the protective role of intensive fat oxidation against diabetes [38, 39]. Thus, despite the positive cross-sectional association with type 2 diabetes and its correlates, intensity of fat oxidation plays a protective role in type 2 diabetes aetiology and should be inversely associated with type 2 diabetes risk.

The intensity of fat oxidation is proportional to circulating NEFA [20, 27, 40-44]. Therefore, we would expect to find similar relationships between fasting NEFA levels and type 2 diabetes risk, i.e. positive cross-sectional and inverse prospective. A recently published study supports this idea. The study compared fasting NEFA 
levels in normal controls, metabolically healthy obese individuals without reduced insulin sensitivity and obese individuals with insulin resistance [45]. Fasting NEFA levels were lower in the normal controls compared with the obese participants, confirming the well-established cross-sectional correlation between fasting NEFA levels and obesity. However, metabolically healthy obese individuals had higher fasting NEFA levels than their insulinresistant counterparts, suggesting that obese individuals with high fasting NEFA levels are less likely to develop type 2 diabetes.

In summary, our results indicate that: (1) $2 \mathrm{~h}$ glucose strongly confounds the prospective association between fasting NEFA and type 2 diabetes; and (2) properly adjusted fasting NEFA levels are inversely associated with risk of type 2 diabetes. The mechanistic explanation of this finding awaits further confirmation.

Acknowledgements We thank C. Byrne (University of Southampton, Southampton, UK) for the interesting discussions and insights into the physiology of fasting NEFA. This research is supported by National Institutes of Health grant 1R01DK081028.

Duality of interest statement The authors declare that there is no duality of interest associated with this manuscript.

\section{References}

1. Boden G (2008) Obesity and free fatty acids. Endocrinol Metab Clin North Am 37:635-6466, viii-ix

2. Arner P (2001) Free fatty acids - do they play a central role in type 2 diabetes? Diabetes Obes Metab 3(Suppl 1):S11-S19

3. Simpson E (1951) The interpretation of interaction in contingency tables. J R Stat Soc 13:238-241

4. Szklo M, Nieto FJ (2007) Identifying noncausal associations: confounding. In: Anonymous epidemiology: beyond the basics, 2nd edn. Jones and Bartlett, Sudbury, pp 151-182

5. Pankow JS, Duncan BB, Schmidt MI et al (2004) Fasting plasma free fatty acids and risk of type 2 diabetes: the Atherosclerosis Risk in Communities Study. Diabetes Care 27:77-82

6. Charles MA, Eschwege E, Thibult N et al (1997) The role of nonesterified fatty acids in the deterioration of glucose tolerance in Caucasian subjects: results of the Paris Prospective Study. Diabetologia 40:1101-1106

7. Paolisso G, Tataranni PA, Foley JE, Bogardus C, Howard BV, Ravussin E (1995) A high concentration of fasting plasma non-esterified fatty acids is a risk factor for the development of NIDDM. Diabetologia 38:1213-1217

8. Byrne CD, Maison P, Halsall D, Martensz N, CN HALES, Wareham NJ (1999) Cross-sectional but not longitudinal associations between non-esterified fatty acid levels and glucose intolerance and other features of the metabolic syndrome. Diabet Med 16:1007-1015

9. Wagenknecht LE, Mayer EJ, Rewers M et al (1995) The Insulin Resistance Atherosclerosis Study (IRAS): objectives, design, and recruitment results. Ann Epidemiol 5:464-472

10. Bergman RN, Finegood DT, Ader M (1985) Assessment of insulin sensitivity in vivo. Endocr Rev 6:45-86
11. Welch S, Gebhart SS, Bergman RN, Phillips LS (1990) Minimal model analysis of intravenous glucose tolerance test-derived insulin sensitivity in diabetic subjects. J Clin Endocrinol Metab 71:1508-1518

12. Steil GM, Volund A, Kahn SE, Bergman RN (1993) Reduced sample number for calculation of insulin sensitivity and glucose effectiveness from the minimal model. Suitability for use in population studies. Diabetes 42:250-256

13. Pacini G, Bergman RN (1986) MINMOD: a computer program to calculate insulin sensitivity and pancreatic responsivity from the frequently sampled intravenous glucose tolerance test. Comput Methods Programs Biomed 23:113-122

14. Saad MF, Anderson RL, Laws A et al (1994) A comparison between the minimal model and the glucose clamp in the assessment of insulin sensitivity across the spectrum of glucose tolerance. Insulin Resistance Atherosclerosis Study. Diabetes 43:1114-1121

15. Korytkowski MT, Berga SL, Horwitz MJ (1995) Comparison of the minimal model and the hyperglycemic clamp for measuring insulin sensitivity and acute insulin response to glucose. Metabolism 44:1121-1125

16. Herbert V, Lau KS, Gottlieb CW, Bleicher SJ (1965) Coated charcoal immunoassay of insulin. J Clin Endocrinol Metab 25:1375-1384

17. Noma A, Okabe H, Kita M (1973) A new colorimetric microdetermination of free fatty acids in serum. Clin Chim Acta 43:317-320

18. Mickey RM, Greenland S (1989) The impact of confounder selection criteria on effect estimation. Am J Epidemiol 129:125-137

19. Hanley AJG, DГÇÖAgostino R, Wagenknecht LE et al (2002) Increased proinsulin levels and decreased acute insulin response independently predict the incidence of type 2 diabetes in the Insulin Resistance Atherosclerosis Study. Diabetes 51:12631270

20. Tataranni PA, Ravussin E (1997) Effect of fat intake on energy balance. Ann N Y Acad Sci 819:37-43

21. Zurlo F, Lillioja S, Esposito-Del Puente A et al (1990) Low ratio of fat to carbohydrate oxidation as predictor of weight gain: study of 24-h RQ. Am J Physiol Endocrinol Metab 259:E650E657

22. Seidell JC, Muller DC, Sorkin JD, Andres R (1992) Fasting respiratory exchange ratio and resting metabolic rate as predictors of weight gain: the Baltimore Longitudinal Study on Aging. Int J Obes Relat Metab Disord 16:667-674

23. Valtuena S, Salas-Salvado J, Lorda PG (1997) The respiratory quotient as a prognostic factor in weight-loss rebound. Int J Obes Relat Metab Disord 21:811-817

24. Marra M, Scalfi L, Contaldo F, Pasanisi F (2004) Fasting respiratory quotient as a predictor of long-term weight changes in non-obese women. Ann Nutr Metab 48:189-192

25. Marra M, Scalfi L, Covino A, Esposito-Del PA, Contaldo F (1998) Fasting respiratory quotient as a predictor of weight changes in non-obese women. Int J Obes Relat Metab Disord 22:601-603

26. Filozof CM, Murua C, Sanchez MP et al (2000) Low plasma leptin concentration and low rates of fat oxidation in weight-stable post-obese subjects. Obes Res 8:205-210

27. Lean ME, James WP (1988) Metabolic effects of isoenergetic nutrient exchange over 24 hours in relation to obesity in women. Int J Obes 12:15-27

28. Buemann B, Astrup A, Madsen J, Christensen NJ (1992) A 24-h energy expenditure study on reduced-obese and nonobese women: effect of beta-blockade. Am J Clin Nutr 56: $662-670$

29. Larson DE, Ferraro RT, Robertson DS, Ravussin E (1995) Energy metabolism in weight-stable postobese individuals. Am J Clin Nutr 62:735-739 
30. Ranneries C, Bulow J, Buemann B, Christensen NJ, Madsen J, Astrup A (1998) Fat metabolism in formerly obese women. Am J Physiol 274:E155-E161

31. Kelley DE, Simoneau JA (1994) Impaired free fatty acid utilization by skeletal muscle in non-insulin-dependent diabetes mellitus. J Clin Invest 94:2349-2356

32. Blaak EE, van Aggel-Leijssen DP, Wagenmakers AJ, Saris WH, van Baak MA (2000) Impaired oxidation of plasma-derived fatty acids in type 2 diabetic subjects during moderate-intensity exercise. Diabetes 49:2102-2107

33. Blaak EE, Wagenmakers AJM, Glatz JFC et al (2000) Plasma FFA utilization and fatty acid-binding protein content are diminished in type 2 diabetic muscle. Am J Physiol Endocrinol Metab 279:E146-E154

34. Lattuada G, Costantino F, Caumo A et al (2005) Reduced wholebody lipid oxidation is associated with insulin resistance, but not with intramyocellular lipid content in offspring of type 2 diabetic patients. Diabetologia 48:741-747

35. Hurley BF, Nemeth PM, Martin WH III, Hagberg JM, Dalsky GP, Holloszy JO (1986) Muscle triglyceride utilization during exercise: effect of training. J Appl Physiol 60:562-567

36. Turcotte LP, Richter EA, Kiens B (1992) Increased plasma FFA uptake and oxidation during prolonged exercise in trained vs untrained humans. Am J Physiol 262:E791-E799

37. Poehlman ET, Melby C (1998) Resistance training and energy balance. Int J Sport Nutr 8:143-159
38. Kelley DE, Goodpaster BH (1999) Effects of physical activity on insulin action and glucose tolerance in obesity. Med Sci Sports Exerc 31:S619-S623

39. Boule NG, Haddad E, Kenny GP, Wells GA, Sigal RJ (2001) Effects of exercise on glycemic control and body mass in type 2 diabetes mellitus: a meta-analysis of controlled clinical trials. JAMA 286:1218-1227

40. Ravussin E, Gautier JF (1999) Metabolic predictors of weight gain. Int J Obes Relat Metab Disord 23(Suppl 1):37-41

41. Schutz Y, Tremblay A, Weinsier RL, Nelson KM (1992) Role of fat oxidation in the long-term stabilization of body weight in obese women. Am J Clin Nutr 55:670-674

42. Groop LC, Bonadonna RC, Shank M, Petrides AS, DeFronzo RA (1991) Role of free fatty acids and insulin in determining free fatty acid and lipid oxidation in man. J Clin Invest 87:8389

43. Groop LC, Bonadonna RC, Simonson DC, Petrides AS, Shank M, DeFronzo RA (1992) Effect of insulin on oxidative and nonoxidative pathways of free fatty acid metabolism in human obesity. Am J Physiol 263:E79-E84

44. Astrup A, Buemann B, Western P, Toubro S, Raben A, Christensen $\mathrm{NJ}$ (1994) Obesity as an adaptation to a high-fat diet: evidence from a cross-sectional study. Am J Clin Nutr 59:350-355

45. Stefan N, Kantartzis K, Machann J et al (2008) Identification and characterization of metabolically benign obesity in humans. Arch InternMed 168:1609-1616 